\title{
Nonparametric adaptive age replacement with a one-cycle criterion
}

\author{
P. Coolen-Schrijner*, F.P.A. Coolen \\ Department of Mathematical Sciences \\ University of Durham, Durham, DH1 3LE, UK \\ *e-mail: Pauline.Schrijner@durham.ac.uk
}

\begin{abstract}
Age replacement of technical units has received much attention in the reliability literature over the last four decades. Mostly, the failure time distribution for the units is assumed to be known, and minimal costs per unit of time is used as optimality criterion, where renewal reward theory simplifies the mathematics involved but requires the assumption that the same process and replacement strategy continues over a very large ('infinite') period of time. Recently, there has been increasing attention to adaptive strategies for age replacement, taking into account the information from the process. Although renewal reward theory can still be used to provide an intuitively and mathematically attractive optimality criterion, it is more logical to use minimal costs per unit of time over a single cycle as optimality criterion for adaptive age replacement. In this paper, we first show that in the classical age replacement setting, with known failure time distribution with increasing hazard rate, the one-cycle criterion leads to earlier replacement than the renewal reward criterion. Thereafter, we present adaptive age replacement with a one-cycle criterion within the nonparametric predictive inferential framework. We study the performance of this approach via simulations, which are also used for comparisons with the use of the renewal reward criterion within the same statistical framework.
\end{abstract}

Key words: Age replacement; nonparametric predictive inference; one-cycle optimality criterion; renewal reward theorem. 


\section{Introduction}

Age replacement strategies for technical units are aimed at replacing such units preventively before failure occurs, trying to avoid the need for corrective replacement which tends to be far more expensive. This requires determination of an optimal preventive replacement time $T>0$, such that the unit will be replaced preventively if it reaches age $T$, or correctively if failure occurs before it reaches age $T$. In the classical stochastic literature [1, 2], age replacement is presented within a traditional Operational Research framework, with the failure time distribution of the unit assumed to be known. If one aims at optimisation in the sense of minimal costs per unit of time, over a very long period of time during which the process considered does not change, and the units used all have the same failure time distribution, then renewal reward theory [1] provides a mathematically convenient way of computing the expected costs per unit of time. In practice, even though one acknowledges the fact that assuming such a long period for the same process may not be realistic, the renewal reward optimality criterion is still often considered attractive and reasonable, with benefit of its mathematical simplicity.

During the last decade $[3,4,5]$, researchers have become interested in so-called adaptive replacement strategies, where instead of assuming complete knowledge of a unit's failure time distribution, one uses information from the process to learn about this distribution. For example, the Bayesian statistical framework $[3,4,5]$ allows the use of a parametric distribution, with an assumed prior distribution reflecting subjective information available before the process starts, and which, combined with process information, gives a posterior distribution, which can be used to determine the optimal replacement strategy for the next unit in the process. As discussed by Mazzuchi and Soyer [3], such an adaptive approach undermines the use of the renewal reward criterion as one explicitly does not wish to use the same replacement strategy over a very long period of time. Hence, they proposed the use of a 'one-cycle' optimality criterion, aiming at minimal expected costs per unit of time for the cycle corresponding to the unit considered.

Throughout this paper, the term 'cycle' is used for the period starting at the moment that a new unit starts functioning, and ending at the moment that the next unit has been installed, following either preventive or corrective replacement. The time required for any replacement is assumed to be neglectible throughout (adapting to include a known replacement time is relatively straightforward), and the costs of preventive replacement and corrective replacement are assumed to be known constants, where the 'costs per cycle' include the costs 
incurred by the replacement at the end of a cycle.

Recently, we have introduced and studied the use of nonparametric predictive inference (NPI) for adaptive age replacement strategies [6, 7], leading to optimal strategies which are fully adaptive to process data without the need to assume a parametric probability distribution for a unit's failure time. So far, we studied NPI-based age replacement using the renewal reward criterion, in this paper we follow Mazzuchi and Soyer's approach by adopting the one-cycle optimality criterion.

For a detailed introduction to NPI in reliability we refer to Coolen, et al. [8], here we briefly give the main concept. Consider the situation where we have observed $n$ failure times for units in the same process, ordered as $0<x_{(1)}<x_{(2)}<\ldots<x_{(n)}$ (we assume throughout this paper that there are no ties in the observed failure times). Instead of assuming a known probability distribution for the failure time $X_{n+1}$ of the next unit, we directly specify probabilities for $X_{n+1}$ according to Hill's assumption $A_{(n)}[9,10]$, that is,

$$
P\left(X_{n+1} \in\left(x_{(j)}, x_{(j+1)}\right)\right)=\frac{1}{n+1}, \quad j=0, \ldots, n,
$$

where $x_{(0)}=0$ and $x_{(n+1)}=r$, where $r$ is either infinity or a finite upper bound for the support of $X_{n+1}$. For a further discussion of $A_{(n)}$, and historical references which provide justifications of this statistical approach, see $[8,10]$. As a consequence of $(1)$, the predictive survival function for $X_{n+1}$, at the times $x_{(j)}$, is equal to

$$
S_{X_{n+1}}\left(x_{(j)}\right)=\frac{n+1-j}{n+1}, \quad j=0, \ldots, n+1 .
$$

Without further assumptions it is not possible to give a precise value for this survival function at other times, as $A_{(n)}$ assigns probability mass $1 /(n+1)$ to the open intervals created by the observed failure times, but does not put any further restriction on the distribution of the probability mass within these intervals. Straightforward bounds for this survival function can be derived at all times [8], these are upper and lower survival functions within the theory of interval probability [10]. These upper and lower survival functions can themselves be used, without further assumptions, as was e.g. done for our NPI-based age replacement strategies using the renewal reward criterion $[6,7]$. In this paper, we will instead introduce a further assumption on the distribution of the probability mass within these intervals, creating a precise survival function for $X_{n+1}$, as we will explain in Section 3.

In Section 2, we compare the optimal age replacement strategies corresponding to the renewal reward criterion and the one-cycle criterion from the classical stochastic perspective, with the failure time distribution assumed to be known. We will show that, for distributions 
with increasing hazard rates, optimal preventive replacement based on the one-cycle criterion will take place earlier than when based on the renewal reward criterion. As our NPI-based method does not assume increasing hazard rates, this result does not apply automatically for NPI-based age replacement with a one-cycle criterion, as presented in Section 3. In Section 4 we illustrate our method via a small example, including comparison with our NPI-based age replacement using the renewal reward criterion [6, 7]. In Section 5 we present and discuss the results from a simulation study, which provide further insights in our adaptive method. We end the paper with some concluding remarks in Section 6, and an appendix containing the proofs of two lemmas presented in Section 3.

\section{Classical age replacement}

In this section, we consider the classical stochastic model for age replacement [1, 2], where the failure time of the unit is assumed to be an absolutely continuously distributed random quantity $X \geq 0$ with known probability distribution, with cumulative distribution function $F(x)=P(X \leq x)$, probability density function (pdf) $f(x)$, hazard rate $h(x)=f(x) /(1-$ $F(x)$ ), and expected value $E(X)$. To avoid mathematical complications, we assume that $F(0)=0, F(x)>0$ for all $x>0$, and $E(X)<\infty$. The costs included in the age replacement model are assumed to be known constants, with cost of preventive replacement $c_{1}$ and cost of corrective replacement $c_{2}$, where $c_{2}>c_{1}>0$.

The renewal reward criterion minimises the expected costs per unit of time, where the same replacement strategy is assumed to be used over an infinite period of time, applied to a sequence of units whose failure time random quantities all have the same known probability distribution. Application of the renewal reward theorem [1] implies that the cost function, for replacement strategy $T>0$ (i.e. replacement at time $T$, or at failure if that occurs before $T)$, equals the expected costs per cycle divided by the expected length of a cycle,

$$
C_{r}(T)=\frac{c_{1}(1-F(T))+c_{2} F(T)}{\int_{0}^{T}(1-F(x)) d x} .
$$

Let the optimal strategy be denoted by $T_{r}$ (which might be equal to infinity), then the first-order necessary condition for optimality (e.g. [11]) of a finite $T_{r}$ is that it must satisfy

$$
h(T) \int_{0}^{T}(1-F(x)) d x-F(T)=\frac{c_{1}}{c_{2}-c_{1}} .
$$

We define

$$
g_{r}(T)=h(T) \int_{0}^{T}(1-F(x)) d x-F(T) .
$$


Clearly, $g_{r}(T)$ is a continuous function with $g_{r}(0)=0$, and, if $h(\infty)=\lim _{T \rightarrow \infty} h(T)$ exists, then

$$
\lim _{T \rightarrow \infty} g_{r}(T)=h(\infty) E(X)-1 .
$$

If $h(T)$ is monotonously strictly increasing, then $g_{r}(T)$ is also strictly increasing as

$$
\frac{d g_{r}(T)}{d T}=h^{\prime}(T) \int_{0}^{T}(1-F(x)) d x
$$

is positive if $h^{\prime}(T)>0$ for all $T>0$. Hence, for monotonously strictly increasing $h(T)$, the condition $g_{r}(T)=\frac{c_{1}}{c_{2}-c_{1}}$ either has no solution, if $h(\infty) \leq \frac{c_{2}}{\left(c_{2}-c_{1}\right) E(X)}$, in which case one would not replace the unit preventively at a finite time, or it has a unique finite solution $T_{r}$, if $h(\infty)>\frac{c_{2}}{\left(c_{2}-c_{1}\right) E(X)}$.

If there is no finite optimal preventive replacement time, then the cost function obtains its minimal value in the limit,

$$
C_{r}(\infty)=\lim _{T \rightarrow \infty} C_{r}(T)=\frac{c_{2}}{E(X)}
$$

Hence, if there is a finite optimal preventive replacement strategy, the minimal expected costs per unit of time, over an infinite time horizon and based on the renewal reward criterion, is less than $\frac{c_{2}}{E(X)}$.

The one-cycle criterion [3] minimises the expected costs per unit of time for a single cycle. This criterion has been mostly neglected in the literature, which may be caused by the fact that, traditionally, one assumes a known probability distribution for the unit's failure time, in which case it is logical to use the same optimal replacement strategy over many cycles. However, when aiming at adaptive age replacement strategies, where the optimal replacement time can change per cycle, the one-cycle criterion is particularly attractive, as such changing replacement times are explicitly excluded by the underlying justification for use of the renewal reward criterion. Mazzuchi and Soyer [3] proposed this one-cycle criterion for Bayesian adaptive age replacement strategies. The cost function, as function of the random failure time $X$, for replacement strategy $T$, is the costs per unit of time during one cycle,

$$
C_{1}(X, T)= \begin{cases}c_{2} / X & \text { if } X<T, \\ c_{1} / T & \text { if } X \geq T .\end{cases}
$$

To avoid mathematical complexity for one-cycle optimality, we assume that $E(1 / X)$ exists, which is a condition on the failure time distribution for $X$ for values close to 0 (which particularly excludes the use of the Exponential distribution close to 0 in what follows). The 
one-cycle optimality criterion is minimisation of the expected value of $C_{1}(X, T)$ with regard to the probability distribution for $X$,

$$
C_{1}(T)=E\left(C_{1}(X, T)\right)=c_{2} \int_{0}^{T} \frac{1}{x} f(x) d x+\frac{c_{1}}{T}(1-F(T)) .
$$

The optimal strategy $T_{1}$, corresponding to this criterion, might be infinite, but if a finite optimum exists it must satisfy the first-order necessary condition for optimality, which leads to

$$
T h(T)=\frac{c_{1}}{c_{2}-c_{1}}
$$

We define

$$
g_{1}(T)=T h(T)
$$

which is clearly a continuous function with $g_{1}(0)=0$, and which is monotonously strictly increasing with no upper bound for $T \rightarrow \infty$ if $h(x)$ is monotonously strictly increasing. Hence, for failure time distributions with such hazard rates, a unique finite optimal preventive replacement time $T_{1}$ exists according to this one-cycle criterion, and this is derived via $g_{1}\left(T_{1}\right)=\frac{c_{1}}{c_{2}-c_{1}}$. If the hazard rate $h(x)$ is such that $g_{1}(T)<\frac{c_{1}}{c_{2}-c_{1}}$ for all finite $T$, which is unlikely to be the case in relevant replacement situations as it relates to a very strong wear-in effect, then the cost function would have minimal value in the limit,

$$
C_{1}(\infty)=\lim _{T \rightarrow \infty} C_{1}(T)=c_{2} E(1 / X)
$$

Hence, in most situations of interest for possible preventive replacement, the minimal expected costs per unit of time when considered over one cycle is less than $c_{2} E(1 / X)$.

We can now compare the optimal age replacement strategies $T_{r}$ and $T_{1}$, corresponding to the renewal reward criterion and the one-cycle criterion, respectively. If the probability distribution of a unit's absolutely continuously distributed failure time $X>0$ is such that $E(X)$ and $E(1 / X)$ are both finite, and that it has a monotonously strictly increasing hazard rate $h(x)$, then

$$
T_{1}<T_{r}
$$

This follows immediately from the fact that, for all $T>0$,

$$
g_{r}(T)<g_{1}(T)
$$

which easily follows from $\int_{0}^{T}(1-F(x)) d x<T$ and $F(T)>0$, and the fact that these functions are continuous and $g_{r}(0)=g_{1}(0)=0$. Of course, $T_{r}$ can be infinite as discussed 
above. Hence, in the classical stochastic setting with known probability distribution for a unit's failure time, if the unit is subject to wearout in the sense of a strictly increasing hazard rate for its failure time, then the optimal age replacement strategy according to the one-cycle criterion leads to earlier preventive replacement than the optimal strategy according to the renewal reward criterion.

\section{$3 \quad$ NPI age replacement with a one-cycle criterion}

In this section, we present NPI-based adaptive age replacement for unit $n+1$, using the one-cycle criterion, hence aiming at minimisation of the cost function $C_{1}(T)$, as given by (10), for random failure time $X_{n+1}$. This provides an intuitively attractive alternative to NPI-based age replacement using the renewal reward criterion, as presented in $[6,7]$.

In $[6,7]$, we did not add any further assumptions on the probability distribution for $X_{n+1}$ to the inferences which follow directly from $A_{(n)}$, using both the upper and lower survival functions for $X_{n+1}$, leading to two optimal age replacement strategies which could be different. The emphasis there was not on such differences, but on how well these strategies adapt to underlying distributions, which was studied via simulations and turned out to be pretty impressive already for sample sizes of $n=10$, when sampling from Weibull distributions with increasing hazard rate.

If we attempt to follow the same approach in this paper, we run into difficulty when using the lower survival function [8] for $X_{n+1}$, as this puts probability $1 /(n+1)$ adherent to 0 , which implies that the expected value of $1 / X_{n+1}$ is not finite. To overcome this problem, we use a pragmatic solution by assuming, in addition to $A_{(n)}$, a particular manner in which the probability masses $1 /(n+1)$ are divided over the intervals created by the $n$ observed failure times. Of course, this additional assumption will influence the resulting strategies, but this influence will decline with increasing $n$. An advantage of this pragmatic approach is that we only need to study our approach for a single survival function related to $n$ observed failure times, while keeping the $A_{(n)}$ assumption.

In this paper, we assume, for NPI-based age replacement with a one-cycle criterion, in addition to $A_{(n)}$, that the probability masses $1 /(n+1)$ assigned to the open intervals between consecutive observed failure times $x_{(1)}, \ldots, x_{(n)}$ are uniformly distributed per interval. The two end intervals of this partition of the real axis require special attention. The probability mass in the interval beyond $x_{(n)}$ can only be distributed uniformly by assuming a finite end-point $r$ for the support of $X_{n+1}$. Alternatively, we could have assumed a particular 
distribution of the probability mass $1 /(n+1)$ over $\left(x_{(n)}, \infty\right)$. However, from the expected costs $C_{1}(T)$, as presented in Section 2, it is clear that the precise distribution of probability mass beyond preventive replacement age $T$ is irrelevant. In situations where the data indicate that preventive replacement may actually be useful, one expects that the optimal preventive replacement age $T$ is less than the largest observed failure time $x_{(n)}$. Therefore, in this paper we assume a finite $r$ and distribute the probability mass $1 /(n+1)$ uniformly over $\left(x_{(n)}, r\right)$, where of course we always take $r$ larger than $x_{(n)}$. If the optimal replacement time over $(0, r]$ would occur beyond $x_{(n)}$, the data would clearly not support preventive replacement.

For the interval $\left(0, x_{(1)}\right)$, a Uniform distribution of the probability mass $1 /(n+1)$ cannot be assumed together with the one-cycle criterion, as the integral of $f(x) / x$ would still not converge over a small interval immediately to the right of 0 . The same problem would also remain with an Exponential distribution over this interval. Hence, we assume that this probability mass $1 /(n+1)$ is distributed over $\left(0, x_{(1)}\right)$ according to a Gamma distribution with shape parameter 2 , and scale parameter $\alpha$ chosen such that

$$
\int_{0}^{x_{(1)}} \alpha^{2} t e^{-\alpha t} d t=\frac{1}{n+1},
$$

so $\alpha$ is calculated via the equation

$$
\frac{n}{n+1}=e^{-\alpha x_{(1)}}\left(1+\alpha x_{(1)}\right)
$$

The assumption $A_{(n)}$, and these additional assumptions for the distribution of the probability masses $1 /(n+1)$ over the intervals created by the observed failure times, lead to the following specification of the predictive survival function for the failure time $X_{n+1}$ of unit $n+1$ within these intervals, to be used together with the $A_{(n)}$-based values at the observed failure times as given by (2) (with $x_{(n+1)}=r$ as before),

$$
\begin{aligned}
S_{X_{n+1}}(x) & =1-\alpha^{2} \int_{0}^{x} t e^{-\alpha t} d t, \quad x \in\left(0, x_{(1)}\right) \\
S_{X_{n+1}}(x) & =\left(\frac{x_{(j+1)}-x}{x_{(j+1)}-x_{(j)}}\right) S_{X_{n+1}}\left(x_{(j)}\right)+\left(\frac{x-x_{(j)}}{x_{(j+1)}-x_{(j)}}\right) S_{X_{n+1}}\left(x_{(j+1)}\right) \\
& =S_{X_{n+1}}\left(x_{(j+1)}\right)+\frac{1}{n+1}\left(\frac{x_{(j+1)}-x}{x_{(j+1)}-x_{(j)}}\right), \quad x \in\left(x_{(j)}, x_{(j+1)}\right), j=1, \ldots, n .
\end{aligned}
$$

Using this predictive survival function for $X_{n+1}$, and the fact that the pdf $f_{X_{n+1}}$ for $X_{n+1}$ satisfies $f_{X_{n+1}}(x)=-d S_{X_{n+1}}(x) / d x$, we obtain the following expression for the pdf for $X_{n+1}$ 
which we use in our NPI-based age replacement with a one-cycle criterion in this paper,

$$
f_{X_{n+1}}(x)=\left\{\begin{array}{l}
\frac{1}{n+1}\left(\frac{1}{x_{(j+1)}-x_{(j)}}\right), x \in\left(x_{(j)}, x_{(j+1)}\right), j=1, \ldots, n, \\
\alpha^{2} x e^{-\alpha x}, \quad x \in\left(0, x_{(1)}\right) .
\end{array}\right.
$$

With this pdf, the expected costs per unit time for NPI-based age replacement of unit $n+1$, using a one-cycle criterion, and based on ordered observed failure times $x_{(1)}, \ldots, x_{(n)}$, is

$$
C_{\text {one }}(T)=E_{X_{n+1}}\left[C_{1}\left(X_{n+1}, T\right)\right]=\int_{0}^{T} \frac{c_{2}}{x} f_{X_{n+1}}(x) d x+\int_{T}^{\infty} \frac{c_{1}}{T} f_{X_{n+1}}(x) d x .
$$

Throughout this paper, we use the notation $C_{\text {one }}(T)$ for the one-cycle criterion cost function in case of our NPI-based age replacement, with the additional assumptions leading to pdf $f_{X_{n+1}}$ as in (20). Substituting this pdf into (21) yields Lemma 1, the proof is given in the Appendix.

\section{Lemma 1}

a. For $T=x_{(j)}$ and $j=1, \ldots, n+1$, we have

$$
C_{\text {one }}\left(x_{(j)}\right)=c_{2} \alpha\left(1-e^{-\alpha x_{(1)}}\right)+\frac{1}{n+1}\left\{c_{2} \sum_{l=1}^{j-1} \frac{\ln \left(x_{(l+1)}\right)-\ln \left(x_{(l)}\right)}{x_{(l+1)}-x_{(l)}}+c_{1}\left(\frac{n+1-j}{x_{(j)}}\right)\right\} .
$$

b. For $T \in\left(x_{(j)}, x_{(j+1)}\right)$ and $j=1, \ldots, n$, we have

$$
\begin{array}{r}
C_{\text {one }}(T)=c_{2} \alpha\left(1-e^{\left.-\alpha x_{(1)}\right)}+\frac{1}{n+1}\left\{c_{2}\left(\sum_{l=1}^{j-1} \frac{\ln \left(x_{(l+1)}\right)-\ln \left(x_{(l)}\right)}{x_{(l+1)}-x_{(l)}}+\frac{\ln (T)-\ln \left(x_{(j)}\right)}{x_{(j+1)}-x_{(j)}}\right)\right.\right. \\
\left.+\frac{c_{1}}{T}\left(\frac{x_{(j+1)}-T}{x_{(j+1)}-x_{(j)}}+n-j\right)\right\} .
\end{array}
$$

c. For $T \in\left(0, x_{(1)}\right)$, we have

$$
C_{\text {one }}(T)=c_{2} \alpha\left(1-e^{-\alpha T}\right)+\frac{c_{1}}{T} \alpha\left(e^{-\alpha T}\left(T+\frac{1}{\alpha}\right)-e^{-\alpha x_{(1)}}\left(x_{(1)}+\frac{1}{\alpha}\right)\right)+\frac{c_{1}}{T} \frac{n}{n+1} .
$$


It is easy to show that the cost function $C_{\text {one }}(T)$, as given in Lemma 1 , is continuous for all $T$. Lemma 2, of which the proof is also given in the Appendix, uses the fact that if the ratio $c_{2} / c_{1}$ satisfies a certain condition, then $C_{\text {one }}(T)$ is first decreasing and thereafter increasing over $\left(x_{(j)}, x_{(j+1)}\right), j=0, \ldots, n$, so that $C_{\text {one }}(T)$ has a unique local minimum over each interval $\left(x_{(j)}, x_{(j+1)}\right)$.

\section{Lemma 2}

a. For each interval $\left(x_{(j)}, x_{(j+1)}\right), j=1, \ldots, n$, if

$$
\frac{x_{(j+1)}+(n-j)\left(x_{(j+1)}-x_{(j)}\right)}{x_{(j+1)}}<\frac{c_{2}}{c_{1}}<\frac{x_{(j+1)}+(n-j)\left(x_{(j+1)}-x_{(j)}\right)}{x_{(j)}}
$$

then $C_{\text {one }}(T)$ has a local minimum in

$$
\xi_{j}=\frac{c_{1}}{c_{2}}\left(x_{(j+1)}+(n-j)\left(x_{(j+1)}-x_{(j)}\right)\right) .
$$

b. For the interval $\left(0, x_{(1)}\right)$, if

$$
\xi_{0}=\frac{c_{1}+\sqrt{c_{1}\left(4 c_{2}-3 c_{1}\right)}}{2 \alpha\left(c_{2}-c_{1}\right)}<x_{(1)}
$$

then $C_{\text {one }}(T)$ has a local minimum in $\xi_{0}$.

Denote by $T_{j}$ the value of $T$ that minimises $C_{\text {one }}(T)$ over the interval $\left[x_{(j)}, x_{(j+1)}\right]$, that is,

$$
T_{j}=\operatorname{argmin}\left\{C_{\text {one }}(T), T \in\left[x_{(j)}, x_{(j+1)}\right], j=0, \ldots, n\right\} .
$$

As a result of Lemma 2 we now obtain the following analytical expressions for $T_{j}$.

A. Let

$$
a=\frac{x_{(j+1)}+(n-j)\left(x_{(j+1)}-x_{(j)}\right)}{x_{(j+1)}}, \text { and } b=\frac{x_{(j+1)}+(n-j)\left(x_{(j+1)}-x_{(j)}\right)}{x_{(j)}} .
$$

For each interval $\left[x_{(j)}, x_{(j+1)}\right], j=1, \ldots, n$, the value of $T$ that minimises $C_{\text {one }}(T)$ for unit $n+1$ is

$$
T_{j}= \begin{cases}x_{(j)} & \text { if } \frac{c_{2}}{c_{1}} \geq b, \\ x_{(j+1)} & \text { if } \frac{c_{2}}{c_{1}} \leq a, \\ \frac{c_{1}}{c_{2}}\left(x_{(j+1)}+(n-j)\left(x_{(j+1)}-x_{(j)}\right)\right) & \text { if } a<\frac{c_{2}}{c_{1}}<b .\end{cases}
$$


B. For the interval $\left[0, x_{(1)}\right], C_{\text {one }}(T)$ is minimal for

$$
T_{0}= \begin{cases}\frac{c_{1}+\sqrt{c_{1}\left(4 c_{2}-3 c_{1}\right)}}{2 \alpha\left(c_{2}-c_{1}\right)} & \text { if } \frac{c_{1}+\sqrt{c_{1}\left(4 c_{2}-3 c_{1}\right)}}{2 \alpha\left(c_{2}-c_{1}\right)}<x_{(1)}, \\ x_{(1)} & \text { if } \frac{c_{1}+\sqrt{c_{1}\left(4 c_{2}-3 c_{1}\right)}}{2 \alpha\left(c_{2}-c_{1}\right)} \geq x_{(1)} .\end{cases}
$$

As a consequence, the global minimum $T_{\text {one }}^{*}$ of the expected costs per unit time for our NPI-based age replacement with a one-cycle criterion, over the interval $[0, r]$ satisfies

$$
T_{\text {one }}^{*}=\operatorname{argmin}\left\{C_{\text {one }}\left(T_{j}\right), j=0, \ldots, n\right\} .
$$

Computations involved in deriving this $T_{o n e}^{*}$, for given ordered failure times $x_{(1)}, \ldots, x_{(n)}$ and assumed value of $r>x_{(n)}$, are straightforward, as every expression is available analytically, except for computation of the scale parameter $\alpha$ determining the specific Gamma distribution used for the interval $\left(0, x_{(1)}\right)$, which is easily derived numerically from (17).

\section{Example}

Suppose we have observed five failure times: 4, 6, 10,11 and 15. Each preventive replacement $\operatorname{costs} c_{1}=1$, while each corrective replacement costs $c_{2}=10$. Assume that an upper bound for the support of $X_{6}$ is given by $r=25$. We want to find the NPI-based optimal age replacement time for unit 6 in the sense of minimising $C_{\text {one }}(\cdot)$, so based on the one-cycle criterion, and using $A_{(5)}$, together with uniformly distributed probability masses within each of the intervals $\left(x_{(1)}, x_{(2)}\right), \ldots,\left(x_{(5)}, r\right)$, Gamma distributed probability mass within the interval $\left(x_{(0)}, x_{(1)}\right)$, and the observed failure times. Applying (30) and (31) yields the local optima $T_{i}$, and the corresponding values of $C_{\text {one }}\left(T_{i}\right)$, per interval, as given in Table 1 . Hence, the optimal age replacement time is $T_{\text {one }}^{*}=2.153$, with corresponding $C_{\text {one }}\left(T_{\text {one }}^{*}\right)=1.0312$.

We compare this optimal strategy with the optimal age replacement time in case the cost function is based on a renewal argument [6, 7]. Using a renewal argument, the long-run expected costs per unit time, for unit $n+1$, are $C_{r}(T)$ as given by (3), which can also be written as

$$
C_{r}(T)=\frac{c_{2}-S(T)\left(c_{2}-c_{1}\right)}{\int_{0}^{T} S(x) d x} .
$$




\begin{tabular}{|l|ll|}
\cline { 2 - 3 } \multicolumn{1}{c|}{} & \multicolumn{1}{c|}{$T_{i}$} & $C_{\text {one }}\left(T_{i}\right)$ \\
\hline$[0,4]$ & 2.153 & 1.0312 \\
{$[4,6]$} & 4 & 1.1561 \\
{$[6,10]$} & 6 & 1.3968 \\
{$[10,11]$} & 10 & 1.5485 \\
{$[11,15]$} & 11 & 1.6877 \\
{$[15,25]$} & 15 & 1.7977 \\
\hline
\end{tabular}

Table 1: Local optima $T_{i}$ per interval, with corresponding values $C_{\text {one }}\left(T_{i}\right)$

In NPI-based age replacement with the renewal criterion [6,7], we used $A_{(n)}$ without adding further assumptions on the distributions of the probability masses per interval. A lower (upper) bound for the survival function is then obtained by shifting all the probability mass in the interval in which $x$ lies to the left (right) end-point of the interval, see [6] for more details. These lower and upper bounds for the survival function lead to upper and lower bounds, respectively, for the long-run expected costs per unit time, also called the renewal upper and lower cost functions. From [6] we know that, for this example, minimising the renewal upper cost function yields an optimal age replacement time of 4 , with corresponding upper cost function value of 0.75 , while minimising the renewal lower cost function yields an optimal age replacement time of 'just before' 4 (see [6] for further details), with corresponding cost function value of 0.25 . Figure 1 shows the cost function based on a one-cycle criterion, together with the renewal upper and lower cost functions. The optimal age replacement time based on a one-cycle criterion is less than the optimal age replacement times corresponding to both the renewal upper and lower cost functions. This is in line with our result (14), which was proven for the classical situation with the probability distribution for the failure time assumed known, and with increasing hazard rate. Hence, the result (14) does not apply in our NPI-based setting, as we do not add any assumption on the hazard rate in our renewal reward setting. In our simulation study, presented in Section 5, we also consider this comparison in detail. Figure 1 also illustrates that, in this example, the one-cycle cost function lies above the renewal upper and lower cost functions for larger values of $T$. We also consider this further in Section 5 . 


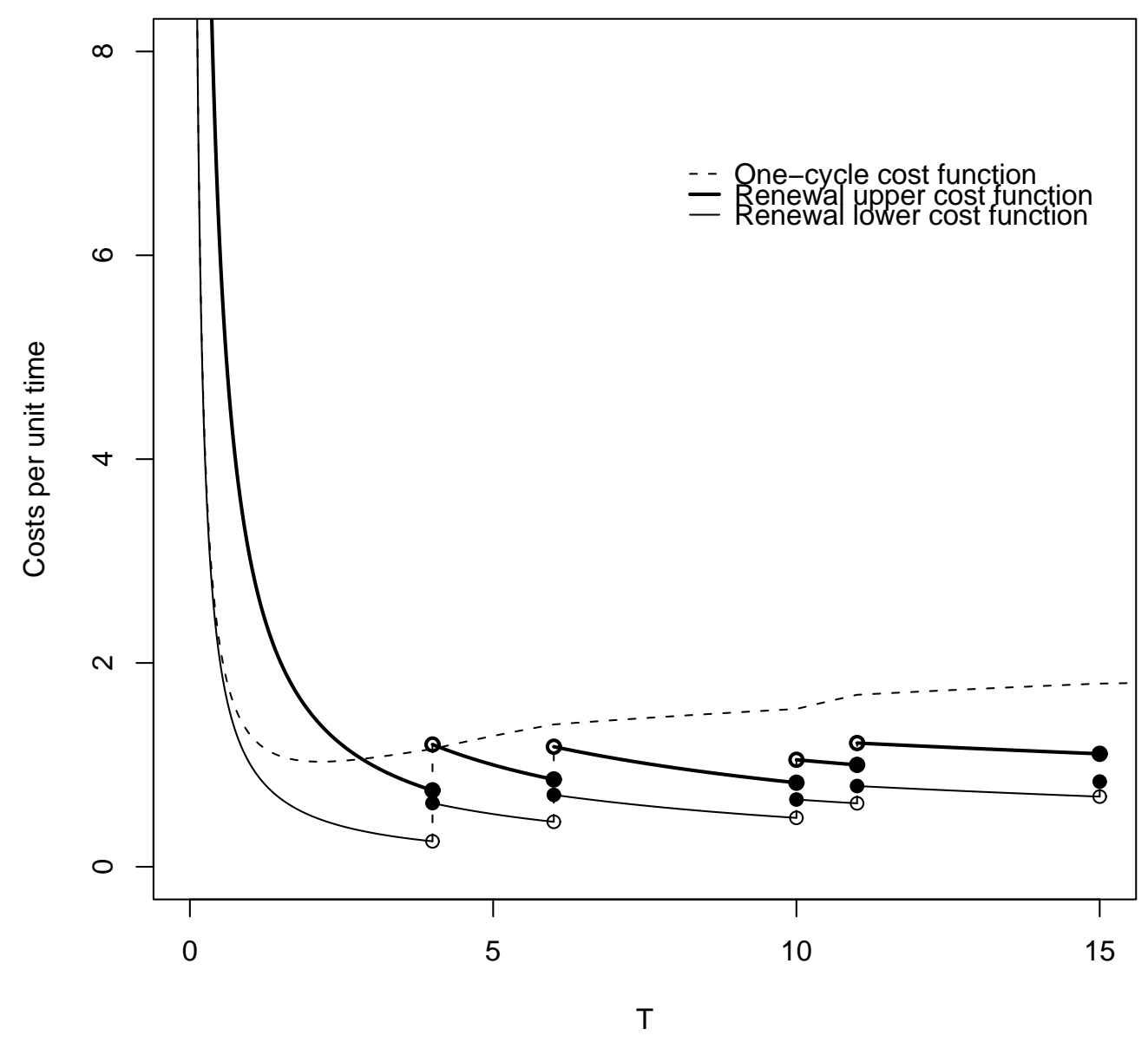

Figure 1: Cost functions for one-cycle and renewal criteria

\section{Simulations}

In this section we present results from simulation studies, to illustrate our NPI-based method and to discuss several of its features. The simulations and computations were performed with the statistical package $R$ [12]. The failure times are simulated from a known probability distribution, where we have restricted attention to the Weibull distribution with scale parameter 1 and shape parameter $\beta$ (denoted by $W(\beta, 1)$ ), which has pdf

$$
f_{X}(t)=\beta t^{\beta-1} e^{\left(-t^{\beta}\right)}, t \geq 0
$$

This enables us to compare our simulation results for the NPI-based optimal replacement times according to the one-cycle criterion, denoted by $T_{\text {one }}^{*}$, with the theoretical optimal 
replacement times according to the same criterion but with the known Weibull distribution, which we denote by $T_{1}^{*}$, and which are obtained by minimisation of the cost function $C_{1}(T)$ as given by (10).

As in the example in Section 4, we also compare our results under the one-cycle criterion with the results under the renewal reward criterion, by calculating the NPI-based optimal replacement times for the renewal lower and upper cost functions, denoted by $\underline{T}_{r e n}^{*}$ and $\bar{T}_{r e n}^{*}$, respectively. We denote by $T_{r}^{*}$ the theoretical optimal age replacement time under the renewal reward criterion for the known probability distribution for $X$, which is obtained by minimisation of the cost function $C_{r}(T)$ as given by (3).

For as far as the replacement costs are concerned, only the ratio $c_{2} / c_{1}$ is relevant for the minimum of the cost function, so we have used $c_{1}=1$, without loss of generality. We have run simulations with $c_{2}$ equal to 10 or 50 . The number of initially observed failure times, $n$, equals 10 or 50, and in each case we have simulated 1000 times. An upper bound $r=5$ for the support of $X_{n+1}$ is assumed, we checked to ensure that all simulated observations where less than 5, which was indeed the case.

Studying the simulation results below, great care must be taken with notation, as we compare two different optimality criteria, for both we consider the NPI-based cost functions and optima as well as the theoretical ones with the Weibull distribution assumed to be known, and we also consider the theoretical cost function values at the NPI-based optimal replacement times, in order to compare the effectiveness of our NPI-based method with the underlying theoretical model. Throughout, we continue to use notation as introduced above. For our NPI-based approach, the one-cycle cost functions and corresponding optimal replacement times have an index 'one', whereas the corresponding NPI-based renewal cost functions and optimal replacement times have an index ' $r e n$ '. The theoretical cost functions and optima, related to the one-cycle criterion, have an index ' 1 ', those related to the renewal criterion have an index ' $r$ '. This leads to the following notation.

The optimal theoretical one-cycle expected age replacement costs per unit time are $C_{1}\left(T_{1}^{*}\right)$, and the optimal theoretical long-run renewal expected costs per unit time are $C_{r}\left(T_{r}^{*}\right)$. Within our NPI-based approach, so explicitly in terms of unit $n+1$, the optimal expected age replacement costs per unit time with the one-cycle criterion is $C_{\text {one }}\left(T_{\text {one }}^{*}\right)$. Using similar notation, $\bar{C}_{r e n}\left(\bar{T}_{r e n}^{*}\right)$ and $\underline{C}_{r e n}\left(\underline{T}_{r e n}^{*}\right)$ are the NPI-based optimal renewal upper and lower long-run expected costs per unit time, respectively. For comparisons in our simulations, we use $\Lambda_{\text {one }}=\left(C_{1}\left(T_{\text {one }}^{*}\right)-C_{1}\left(T_{1}^{*}\right)\right) / C_{1}\left(T_{1}^{*}\right), \bar{\Lambda}_{r e n}=\left(C_{r}\left(\bar{T}_{r e n}^{*}\right)-C_{r}\left(T_{r}^{*}\right)\right) / C_{r}\left(T_{r}^{*}\right)$ and $\underline{\Lambda}_{r e n}=\left(C_{r}\left(\underline{T}_{r e n}^{*}\right)-C_{r}\left(T_{r}^{*}\right)\right) / C_{r}\left(T_{r}^{*}\right)$. These $\Lambda^{\prime} s$ indicate how good our NPI-based optimal 


\begin{tabular}{|l|rr|rr|}
\cline { 2 - 5 } \multicolumn{1}{c|}{} & \multicolumn{2}{c|}{$W(2,1)$} & \multicolumn{2}{c|}{$W(3,1)$} \\
\cline { 2 - 5 } \multicolumn{1}{c|}{} & $c_{2}=10$ & $c_{2}=50$ & $c_{2}=10$ & $c_{2}=50$ \\
\hline$T_{1}^{*}$ & 0.2357 & 0.1010 & 0.3333 & 0.1895 \\
$C_{1}\left(T_{1}^{*}\right)$ & 8.6416 & 19.8663 & 4.5332 & 7.9272 \\
$C_{1}(\infty)$ & 17.7245 & 88.6227 & 13.5412 & 67.7059 \\
$\Lambda_{1}(\infty)$ & 1.0511 & 3.4610 & 1.9871 & 7.5410 \\
$T_{r}^{*}$ & 0.3365 & 0.1431 & 0.3825 & 0.2170 \\
$C_{r}\left(T_{r}^{*}\right)$ & 6.0561 & 14.0239 & 3.9494 & 6.9215 \\
$C_{r}(\infty)$ & 11.2838 & 56.4190 & 11.1985 & 55.9923 \\
$\Lambda_{r}(\infty)$ & 0.8632 & 3.0231 & 1.8355 & 7.0896 \\
\hline
\end{tabular}

Table 2: Theoretical results

replacement times are, compared to the corresponding theoretical optima. Clearly, a low value of such a $\Lambda$ indicates that our method performs well, in the sense that our optimal NPI-based replacement time, which is based directly on the simulated set of failure data, provides a replacement strategy which is nearly as cost effective as the theoretically best strategy, which of course in practice is not known as the underlying failure time distribution will not be known. Hence, these $\Lambda$ 's are useful performance measures for our method, illustrating the joint effects of how well our NPI-based age replacement strategies adapt to the data, and how well the data capture the characteristics of the underlying failure time distribution for as far as relevant to these inferences.

Table 2 gives the theoretical optimal replacement times $T_{1}^{*}$ and $T_{r}^{*}$, and the corresponding minimal cost function values $C_{1}\left(T_{1}^{*}\right)$ and $C_{r}\left(T_{r}^{*}\right)$, for the failure time distributions $W(2,1)$ and $W(3,1)$. We have also included the limiting values of these cost functions for $T \rightarrow \infty$, denoted by $C_{1}(\infty)$ and $C_{r}(\infty)$, which relate to no preventive replacement being carried out, and the values $\Lambda_{1}(\infty)=\left(C_{1}(\infty)-C_{1}\left(T^{*}\right)\right) / C_{1}\left(T^{*}\right)$ and $\Lambda_{r}(\infty)=\left(C_{r}(\infty)-C_{r}\left(T_{r}^{*}\right)\right) / C_{r}\left(T_{r}^{*}\right)$, which are indicators of the theoretical losses, relative to the optimal costs, if no preventive replacements were carried out. The values of $\Lambda_{1}(\infty)$ and $\Lambda_{r}(\infty)$ are larger for $W(3,1)$ than for $W(2,1)$, which indicates that preventive replacement is more effective for $W(3,1)$ than for $W(2,1)$. This results from the fact that the variance of the $W(3,1)$ distribution is smaller than the variance of $W(2,1)$, and replacement policies tend to be more effective if the underlying distribution has a smaller variance [6, 7]. Tables 3 and 4 give the simulation results for $W(2,1)$ and $W(3,1)$, respectively. Tables 5 and 6 show the positions of the 


\begin{tabular}{|c|c|c|c|c|c|c|c|c|c|}
\hline & $T_{\text {one }}^{*}$ & $C_{\text {one }}\left(T_{\text {one }}^{*}\right)$ & $\Lambda_{\text {one }}$ & $\bar{T}_{r e n}^{*}$ & $\bar{C}_{r e n}\left(\bar{T}_{r e n}^{*}\right)$ & $\bar{\Lambda}_{r e n}$ & $\underline{T}_{r e n}^{*}$ & $\underline{C}_{r e n}\left(\underline{T}_{r e n}^{*}\right)$ & $\underline{\Lambda}_{r e n}$ \\
\hline & \multicolumn{9}{|c|}{ CASE 1-1: $W(2,1), c_{2}=10$ and $n=10$} \\
\hline Mean & 0.2669 & 16.2644 & 0.0989 & 0.5454 & 6.5055 & 0.1357 & 0.4793 & 3.7927 & 0.0974 \\
\hline Median & 0.2526 & 10.7120 & 0.0438 & 0.4978 & 6.1994 & 0.0809 & 0.3949 & 3.4985 & 0.0437 \\
\hline \multirow[t]{2}{*}{$\mathrm{Sd}$} & 0.1137 & 48.7599 & 0.1698 & 0.2336 & 2.1377 & 0.1545 & 0.5118 & 94 & 0.1418 \\
\hline & \multicolumn{9}{|c|}{ CASE 1-2: $W(2,1), c_{2}=10$ and $n=50$} \\
\hline Mean & 0.2602 & 11.0495 & 0.0416 & 0.3921 & 5.7470 & 0.0450 & 0.3613 & 5.0962 & 0.0438 \\
\hline Median & 0.2552 & 9.6318 & 0.0188 & 0.3715 & 5.7014 & 0.0214 & 0.3438 & 5.0497 & 0.0213 \\
\hline \multirow[t]{2}{*}{$\mathrm{Sd}$} & 0.0733 & 6.1479 & 0.0598 & 0.1204 & 1.0545 & 0.0605 & 0.1138 & 1.0055 & 0.0579 \\
\hline & \multicolumn{9}{|c|}{ CASE 1-3: $W(2,1), c_{2}=50$ and $n=50$} \\
\hline Mean & 0.1078 & 34.1054 & 0.1334 & 0.2375 & 14.2651 & 0.1651 & 0.1836 & 8.6752 & 0.1020 \\
\hline Median & 0.0999 & 24.4769 & 0.0605 & 0.2244 & 13.4647 & 0.1024 & 0.1737 & 7.9462 & 0.0553 \\
\hline $\mathrm{Sd}$ & 0.0491 & 37.3376 & 0.2226 & 0.0845 & 4.5893 & 0.1808 & 0.0742 & 3.7394 & 0.1265 \\
\hline
\end{tabular}

Table 3: Simulation results for $W(2,1)$

individual values $T_{\text {one }}^{*}$ in relation to the corresponding values $T_{1}^{*}, \bar{T}_{r e n}^{*}$ and $\underline{T}_{r e n}^{*}$.

The main conclusions that can be drawn from these simulation results are discussed below. We also pay attention to the results based on the one-cycle criterion versus those for the renewal criterion. We refer to $[6,7]$ for conclusions on such results from similar simulation studies for the renewal criterion only.

\section{$\underline{\text { Conclusions from simulations: }}$}

1. Tables 3 and 4 show that the means and medians of $T_{\text {one }}^{*}$ are smaller than the means and medians of $\bar{T}_{r e n}^{*}$ and $\underline{T}_{r e n}^{*}$, which is in line with result (14) (even though, as discussed before, (14) does not apply here). Tables 5 and 6 show that this not only holds for the means and medians, but for all individual simulated data sets in our study, although in several situations the optima according to the different criteria are equal (see point 7 below). The standard deviations of $T_{\text {one }}^{*}$ are also smaller than the corresponding ones for the renewal criterion, indicating less spread in the optima, in particular fewer relatively large values occur when the one-cycle criterion is used. 


\begin{tabular}{|c|c|c|c|c|c|c|c|c|c|}
\hline & $T_{\text {one }}^{*}$ & $C_{\text {one }}\left(T_{\text {one }}^{*}\right)$ & $\Lambda_{\text {one }}$ & $\bar{T}_{r e n}^{*}$ & $\bar{C}_{r e n}\left(\bar{T}_{r e n}^{*}\right)$ & $\bar{\Lambda}_{r e n}$ & $\underline{T}_{r e n}^{*}$ & $\underline{C}_{r e n}\left(\underline{T}_{r e n}^{*}\right)$ & $\underline{\Lambda}_{r e n}$ \\
\hline & \multicolumn{9}{|c|}{ CASE 2-1: $W(3,1), c_{2}=10$ and $n=10$} \\
\hline Mean & 0.3484 & 8.1485 & 0.1225 & 0.5475 & 4.8295 & 0.2064 & 0.4843 & 2.6125 & 0.1529 \\
\hline Median & 0.3397 & 6.9477 & 0.0609 & 0.5429 & 4.6075 & 0.1343 & 0.4764 & 2.4340 & 0.0888 \\
\hline \multirow[t]{2}{*}{$\mathrm{Sd}$} & 0.1160 & 4.7086 & 0.1850 & 0.1527 & 1.4773 & 0.2271 & 0.1518 & 0.9619 & 0.1814 \\
\hline & \multicolumn{9}{|c|}{ CASE 2-2: $W(3,1), c_{2}=10$ and $n=50$} \\
\hline Mean & 0.3660 & 5.6566 & 0.0565 & 0.4338 & 3.8672 & 0.0589 & 0.4139 & 3.3477 & 0.0535 \\
\hline Median & 0.3577 & 5.2749 & 0.0249 & 0.4297 & 3.8237 & 0.0315 & 0.4037 & 3.2960 & 0.0276 \\
\hline \multirow[t]{2}{*}{$\mathrm{Sd}$} & 0.0814 & 1.9090 & 0.0772 & 0.0919 & 0.6792 & 0.0741 & 0.0920 & 0.6261 & 0.0685 \\
\hline & \multicolumn{9}{|c|}{ CASE 2-3: $W(3,1), c_{2}=50$ and $n=50$} \\
\hline Mean & 0.1815 & 14.6619 & 0.1356 & 0.3080 & 8.3397 & 0.2185 & 0.2723 & 4.5780 & 0.1590 \\
\hline Median & 0.1784 & 12.2366 & 0.0558 & 0.2995 & 8.0505 & 0.1185 & 0.2670 & 4.2240 & 0.0799 \\
\hline $\mathrm{Sd}$ & 0.0613 & 9.1017 & 0.2515 & 0.0819 & 2.3185 & 0.2563 & 0.0835 & 1.6400 & 0.2036 \\
\hline
\end{tabular}

Table 4: Simulation results for $W(3,1)$

2. Tables 3 and 4 , when compared to Table 2, show that the means and medians of $T_{\text {one }}^{*}$ are pretty close to the corresponding $T_{1}^{*}$, which indicates that our method adapts well to the underlying distribution for data sets of size 10 and 50, as used in this simulation study. For the corresponding values based on the renewal criterion, there is a noticeable improvement in the adaptation to the underlying distribution for $n=50$ over $n=10$. From this study, it seems that our NPI-based method adapts better to the underlying distribution for the one-cycle criterion than for the renewal criterion, in particular for smaller values of $n$ (see also points 5 and 6 below). Tables 5 and 6 show that, for individual cases, $T_{\text {one }}^{*}$ can be smaller or larger than the theoretical $T_{1}^{*}$ based on the underlying failure time distribution. This is caused by our NPI-based method adapting well to the sampled data, and the randomness in such simulated data.

3. The means and medians of $C_{\text {one }}\left(T_{\text {one }}^{*}\right)$ are larger than $C_{1}\left(T_{1}^{*}\right)$. However, for individual cases (not shown), $C_{\text {one }}\left(T_{\text {one }}^{*}\right)$ can also be smaller than $C_{1}\left(T_{1}^{*}\right)$. The means and medians of $C_{\text {one }}\left(T_{o n e}^{*}\right)$ are also larger than the corresponding values of $\bar{C}_{r e n}\left(\bar{T}_{r e n}^{*}\right), \underline{C}_{r e n}\left(\underline{T}_{r e n}^{*}\right)$, and $C_{r}\left(T_{r}^{*}\right)$. 


\begin{tabular}{|c|rrr||rrr||rrr|}
\cline { 2 - 9 } \multicolumn{1}{c|}{} & \multicolumn{3}{c||}{ Case 1-1 } & \multicolumn{3}{c||}{ Case 1-2 } & \multicolumn{3}{c|}{ Case 1-3 } \\
\cline { 2 - 9 } \multicolumn{1}{c|}{} & $T_{1}^{*}$ & $\bar{T}_{\text {ren }}^{*}$ & $\underline{T}_{r e n}^{*}$ & $T_{1}^{*}$ & $\bar{T}_{r e n}^{*}$ & $\underline{T}_{r e n}^{*}$ & $T_{1}^{*}$ & $\bar{T}_{r e n}^{*}$ & $\underline{T}_{r e n}^{*}$ \\
\hline$T_{\text {one }}^{*}>$ & 562 & 0 & 0 & 607 & 0 & 0 & 495 & 0 & 0 \\
$T_{\text {one }}^{*}=$ & 0 & 0 & 0 & 0 & 324 & 451 & 0 & 0 & 0 \\
$T_{\text {one }}^{*}<$ & 438 & 1000 & 1000 & 393 & 676 & 549 & 505 & 1000 & 1000 \\
\hline
\end{tabular}

Table 5: The positions of the NPI-based $T_{\text {one }}^{*}$ for $\mathrm{W}(2,1)$

\begin{tabular}{|c|rrr||rrr||rrr|}
\cline { 2 - 9 } \multicolumn{1}{c|}{} & \multicolumn{3}{c||}{ Case 2-1 } & \multicolumn{3}{c||}{ Case 2-2 } & \multicolumn{3}{c|}{ Case 2-3 } \\
\cline { 2 - 3 } \multicolumn{1}{c|}{} & $T^{*}$ & $\bar{T}_{\text {ren }}^{*}$ & $\underline{T}_{r e n}^{*}$ & $T^{*}$ & $\bar{T}_{r e n}^{*}$ & $\underline{T}_{r e n}^{*}$ & $T^{*}$ & $\bar{T}_{r e n}^{*}$ & $\underline{T}_{r e n}^{*}$ \\
\hline$T_{\text {one }}^{*}>$ & 514 & 0 & 0 & 632 & 0 & 0 & 437 & 0 & 0 \\
$T_{\text {one }}^{*}=$ & 0 & 0 & 0 & 0 & 562 & 676 & 0 & 0 & 0 \\
$T_{\text {one }}^{*}<$ & 486 & 1000 & 1000 & 368 & 438 & 324 & 563 & 1000 & 1000 \\
\hline
\end{tabular}

Table 6: The positions of the NPI-based $T_{\text {one }}^{*}$ for $\mathrm{W}(3,1)$

4. The medians of $T_{\text {one }}^{*}$ are smaller than the corresponding means, which is due to the fact that the distributions of the $T_{\text {one }}^{*}$ 's are skewed to the right. In our earlier work on NPI-based age replacement with the renewal criterion $[6,7]$, this was also discussed, and we regard the medians as better indicators of the performance of our methods than the means.

5. The means and medians of $\Lambda_{\text {one }}$ are pretty small, indicating that our method tends to adapt well to the data, in the sense that the resulting NPI-based optimal replacement times would not lead to large losses compared to the theoretically optimal replacement times. The distributions of the values $\Lambda_{\text {one }}$ from our simulations are highly skewed to the right, meaning that many values are very close to 0 , but some are large (which is shown both by the large differences between corresponding means and medians, and the large standard deviations). Due to the nature of the one-cycle cost function, large values of $\Lambda_{\text {one }}$ occur particularly if the NPI-based method suggests relatively small preventive replacement times, which occurs if the simulated data happens to include small failure times. For increasing $n$ (compare Cases 1-1 and 1-2, and Cases 2-1 and 22 ), the $\Lambda_{\text {one }}$ tend to decrease, which shows that our method provides better adaptation to the underlying distribution for larger values of $n$. In particular, for larger $n$ the effect 
of very small simulated data values decreases, so the NPI-based preventive replacement times do not become very small as easily as for $n=10$.

6. Comparing Cases 1-2 and 1-3, and Cases 2-2 and 2-3, we see that the means and medians of $\Lambda_{\text {one }}$ increase with $c_{2}$, and also the standard deviations increase noticeably. For larger corrective replacement costs, one is more careful and tends to plan earlier preventive replacement. Therefore, if the simulated data include one or more very small observed failure times, the NPI-based age replacement time is also very small, leading to large $\Lambda_{\text {one }}$.

7. If $n$ is small or $c_{2} / c_{1}$ is large (Cases $1-1,1-3,2-1$ and 2-3), $T_{\text {one }}^{*}$ is smaller than $\bar{T}_{\text {ren }}^{*}$ and $\underline{T}_{\text {ren }}^{*}$ (see Tables 5 and 6 ). However, if $n$ is not too small and $c_{2} / c_{1}$ not too large, $T_{\text {one }}^{*}$ is quite often equal to $\bar{T}_{r e n}^{*}$ or $\underline{T}_{r e n}^{*}$ (Cases 1-2 and 2-2). This follows from (30) and (31) and the fact that the renewal upper and lower cost functions are minimised in the right end-point of each interval (or 'just before' it, see [6]).

\section{Concluding Remarks}

The method presented in this paper is fully adaptive to available failure data, but therefore requires availability of such data, which may often be unrealistic. However, one should not forget that the classical method assumes full knowledge of an underlying failure time distribution, which is even more far stretched. Of course, if such failure data are available, our method is of direct use, either on its own or, which may be more attractive, in combination with more established methods for age replacement [1] or alternative adaptive methods $[3,4,5]$. If different methods result in pretty similar preventive replacement times, the data strongly support the additional assumptions underlying the other methods, and it is straightforward to decide on a good replacement policy. If, however, these different methods lead to rather different optimal replacement times, our method has the advantage of being based on quite minimal modelling assumptions, so the resulting strategies from the other models are more influenced by modelling assumptions, which one should then try to verify or study in more detail. Our method can also be generalized, relatively straightforwardly, to situations where the observed data include some right-censored observations, which may for example result from earlier preventive replacements. This can be achieved by replacing $A_{(n)}$ by the generalized assumption presented by Coolen and Yan [13], which similarly provides predictive probabilities for the failure time of the next unit based on such data. 
A key motivation for this work, as alternative to our recent NPI-methods for age replacement based on the renewal criterion [6,7], is the natural use of the one-cycle criterion for fully adaptive methods, with the idea that the replacement time for unit $n+2$ will also adapt to the information that we would get on unit $n+1$ within the process. We have not studied this adaptation for further future units in this paper, but we did consider it explicitly in [7] for the renewal criterion. Clearly, for smaller $n$ the extra information from unit $n+1$ has relatively more influence on the optimal replacement time for unit $n+2$ than for larger values of $n$. As far as we are aware, no detailed comparisons of different optimality criteria for age replacement have been published. In practice, it may not be precisely clear which criterion is most suitable, there may well be other criteria that fit better with some particular applications. In addition to direct comparisons of optimal replacement times according to different criteria, as presented in Sections 2 and 5, it is also of interest to study robustness of replacement strategies with regard to different optimality criteria, both within NPI and more generally.

Of course, in the literature many variations to age replacement have been studied, and many of these can also be studied from NPI perspective. One such variation is so-called 'opportunity-based age replacement' [14], where it is acknowledged that one may not be able to plan preventive replacement at any moment, but can only perform such replacement at randomly occurring opportunities. In [15] we studied this from NPI perspective, restricting to the renewal criterion, where again the methods seem to provide good adaptation to the failure data. 


\section{Appendix}

\section{$\underline{\text { Proof of Lemma } 1}$}

a. For $T=x_{(j)}$ and $j=1, \ldots, n+1$, we have

$$
\begin{aligned}
C_{\text {one }}\left(x_{(j)}\right) & =\int_{0}^{x_{(1)}} \frac{c_{2}}{x} f_{X_{n+1}}(x) d x+\sum_{l=1}^{j-1} \int_{x_{(l)}}^{x_{(l+1)}} \frac{c_{2}}{x} f_{X_{n+1}}(x) d x+\frac{c_{1}}{T} \int_{x_{(j)}}^{r} f_{X_{n+1}}(x) d x \\
& =c_{2} \int_{0}^{x_{(1)}} \frac{1}{x} \alpha^{2} x e^{-\alpha x} d x+\sum_{l=1}^{j-1} \int_{x_{(l)}}^{x_{(l+1)}} \frac{c_{2}}{x} \frac{1}{(n+1)} \frac{1}{\left(x_{(l+1)}-x_{(l)}\right)} d x+\frac{c_{1}}{T}\left(\frac{n+1-j}{n+1}\right) \\
& =c_{2} \int_{0}^{x_{(1)}} \alpha^{2} e^{-\alpha x} d x+\sum_{l=1}^{j-1} \frac{x_{(l+1)}}{(n+1)\left(x_{(l+1)}-x_{(l)}\right)} \int_{x_{(l)}}^{\frac{1}{x}} d x+\frac{c_{1}}{x_{(j)}}\left(\frac{n+1-j}{n+1}\right) \\
& =c_{2} \alpha\left(1-e^{-\alpha x(1)}\right)+\frac{1}{n+1}\left\{c_{2} \sum_{l=1}^{j-1} \frac{\ln \left(x_{(l+1)}\right)-\ln \left(x_{(l)}\right)}{x_{(l+1)}-x_{(l)}}+c_{1}\left(\frac{n+1-j}{x_{(j)}}\right)\right\} .(35)
\end{aligned}
$$

b. For $T \in\left(x_{(j)}, x_{(j+1)}\right)$ and $j=1, \ldots, n$, substituting (20) into

$$
\begin{aligned}
C_{\text {one }}(T)= & \int_{0}^{x_{(1)}} \frac{c_{2}}{x} f_{X_{n+1}}(x) d x+\sum_{l=1}^{j-1} \int_{x_{(l)}}^{x_{(l+1)}} \frac{c_{2}}{x} f_{X_{n+1}}(x) d x+\int_{x_{(j)}}^{T} \frac{c_{2}}{x} f_{X_{n+1}}(x) d x \\
& +\int_{T}^{x_{(j+1)}} \frac{c_{1}}{T} f_{X_{n+1}}(x) d x+\int_{x_{(j+1)}}^{r} \frac{c_{1}}{T} f_{X_{n+1}}(x) d x
\end{aligned}
$$

yields the expression given in Lemma $1 \mathrm{~b}$.

c. For $T \in\left(0, x_{(1)}\right)$, substituting (20) into

$$
C_{\text {one }}(T)=\int_{0}^{T} \frac{c_{2}}{x} f_{X_{n+1}}(x) d x+\int_{T}^{x_{(1)}} \frac{c_{1}}{T} f_{X_{n+1}}(x) d x+\int_{x_{(1)}}^{r} \frac{c_{1}}{T} f_{X_{n+1}}(x) d x
$$

yields the expression given in Lemma 1c. 
a. For $T \in\left(x_{(j)}, x_{(j+1)}\right), j=1, \ldots, n$, we have

$$
\begin{aligned}
\frac{d C_{\text {one }}(T)}{d T} & =\frac{1}{n+1}\left[\frac{c_{2}}{T\left(x_{(j+1)}-x_{(j)}\right)}-\frac{c_{1}}{T^{2}}\left(\frac{x_{(j+1)}-T}{x_{(j+1)}-x_{(j)}}+n-j\right)-\frac{c_{1}}{T\left(x_{(j+1)}-x_{(j)}\right)}\right] \\
& =\frac{1}{(n+1) T\left(x_{(j+1)}-x_{(j)}\right)}\left[c_{2}-\frac{c_{1} x_{(j+1)}}{T}-\frac{c_{1}(n-j)\left(x_{(j+1)}-x_{(j)}\right)}{T}\right]
\end{aligned}
$$

Hence, we have that $\left.\frac{d C_{\text {one }}(T)}{d T}\right|_{T=\xi_{j}}=0$ where

$$
\xi_{j}=\frac{c_{1}}{c_{2}}\left(x_{(j+1)}+(n-j)\left(x_{(j+1)}-x_{(j)}\right)\right)
$$

while $\frac{d C_{\text {one }}(T)}{d T}<0$ for $T \in\left(x_{(j)}, \xi_{j}\right)$ and $\frac{d C_{\text {one }}(T)}{d T}>0$ for $T \in\left(\xi_{j}, x_{(j+1)}\right)$ as long as

$$
x_{(j)}<\frac{c_{1}}{c_{2}}\left(x_{(j+1)}+(n-j)\left(x_{(j+1)}-x_{(j)}\right)\right)<x_{(j+1)},
$$

that is, as long as the ratio $c_{2} / c_{1}$ satisfies the condition given in Lemma $2 \mathrm{a}$.

b. For $T \in\left(0, x_{(1)}\right)$, we have

$$
\begin{aligned}
\frac{d C_{\text {one }}(T)}{d T}= & c_{2} \alpha^{2} e^{-\alpha T}-c_{1} \alpha^{2} e^{-\alpha T}-\frac{c_{1}}{T^{2}} e^{-\alpha T}-\alpha \frac{c_{1}}{T} e^{-\alpha T}+\frac{c_{1}}{T^{2}} \alpha e^{-\alpha x_{(1)}}\left(x_{(1)}+\frac{1}{\alpha}\right) \\
& -\frac{c_{1}}{T^{2}} \frac{n}{n+1} \\
= & e^{-\alpha T}\left(\left(c_{2}-c_{1}\right) \alpha^{2}-\frac{c_{1}}{T^{2}}-\frac{c_{1}}{T} \alpha\right)
\end{aligned}
$$

where we have used the fact that $\alpha$ has been chosen such that it satisfies (17). Hence, we have that $\left.\frac{d C_{\text {one }}(T)}{d T}\right|_{T=\xi_{0}}=0$ where

$$
\xi_{0}=\frac{c_{1}+\sqrt{c_{1}\left(4 c_{2}-3 c_{1}\right)}}{2 \alpha\left(c_{2}-c_{1}\right)}
$$

while $\frac{d C_{\text {one }}(T)}{d T}<0$ for $T \in\left(0, \xi_{0}\right)$ and $\frac{d C_{\text {one }}(T)}{d T}>0$ for $T \in\left(\xi_{0}, x_{(1)}\right)$ as long as $0<\xi_{0}<x_{(1)}$.

\section{Acknowledgement}

This research is supported by the UK Engineering and Physical Research Sciences Council, grant GR/R92530/01. 


\section{References}

[1] Barlow, R.E. and Proschan, F. (1965). Mathematical Theory of Reliability. Wiley, New York.

[2] Aven, T. and Jensen, U. (1999). Stochastic Models in Reliability. Springer, New York.

[3] Mazzuchi, T.A. and Soyer, R. (1996). A Bayesian perspective on some replacement strategies. Reliability Engineering and System Safety, 51, 295-303.

[4] Sheu, S.H., Yeh, R.H., Lin, Y.B. and Juan, M.G. (1999). A Bayesian perspective on age replacement with minimal repair. Reliability Engineering and System Safety, 65, 55-64.

[5] Sheu, S.H., Yeh, R.H., Lin, Y.B. and Juan, M.G. (2001). A Bayesian approach to an adaptive preventive maintenance model. Reliability Engineering and System Safety, 71, 33-44.

[6] Coolen-Schrijner, P. and Coolen, F.P.A. (2004a). Non-parametric predictive inference for age replacement with a renewal argument. Quality and Reliablity Engineering International, 20, 203-215.

[7] Coolen-Schrijner, P. and Coolen, F.P.A. (2004b). Adaptive age replacement strategies based on nonparametric predictive inference. Journal of the Operational Research Society, to appear.

[8] Coolen, F.P.A., Coolen-Schrijner, P. and Yan, K.J. (2002). Nonparametric predictive inference in reliability. Reliability Engineering and System Safety, 78, 185-193.

[9] Hill, B.M. (1968). Posterior distribution of percentiles: Bayes' theorem for sampling from a population. Journal of the American Statistical Association, 63, 677-691.

[10] Augustin, T. and Coolen, F.P.A. (2004). Nonparametric predictive inference and interval probability. Journal of Statistical Planning and Inference, 124, 251-272.

[11] Luenberger, D.G. (1973). Introduction to Linear and Nonlinear Programming. AddisonWesley, Menlo Park.

[12] The R Project for Statistical Computing: www.r-project.org.

[13] Coolen, F.P.A. and Yan, K.J. (2004). Nonparametric predictive inference with rightcensored data. Journal of Statistical Planning and Inference, 126, 25-54. 
[14] Dekker, R. and Dijkstra, M.C. (1992). Opportunity-based age replacement: Exponentially distributed times between opportunities. Naval Research Logistics, 39, 175-190.

[15] Coolen-Schrijner, P., Coolen, F.P.A. and Shaw, S.C. (2004). Nonparametric adaptive opportunity-based age replacement strategies. In submission. 